\title{
Economic perspectives on the European Neighbourhood Policy. An introduction
}

\author{
Ron Boschma*, Simona Iammarino\#, Raffaele Paci\$ and Jordi Suriñach+ \\ *Utrecht University \\ \#London School of Economics \\ \$University of Cagliari \\ +AQR-IREA. University of Barcelona
}

special issue

Journal of Economic and Social Geography

\section{ENP}

In the past, the European Union (EU) established partnership agreements to strengthen cooperation with its neighbouring countries. In 2004, the European Neighbourhood Policy (ENP) was put in place with the objectives of avoiding the emergence of new frontier divisions between the enlarged EU and its immediate neighbours, and at the same time creating the conditions to strengthen peace, prosperity and stability to all countries involved. The ENP has turned into a unified policy framework for the EU's neighbours, offering preferential economic and political relations in exchange for the recipient countries' adherence to the Union's principles. The ultimate aim of the ENP is to strengthen the prosperity, stability and security of the EU by creating a "ring of friends" around the EU's political border.

However, there is surprisingly little research on ENP (Suriñach 2015). Until now, empirical studies on ENP are scarce and the majority of those with an economic perspective are focused on trade. Migration or investment flows received far less attention. Moreover, only a few studies have examined innovation in the European Neighbouring Countries (ENCs), and no contribution explicitly examined the role of the institutional environment, cultural diversity and the effects of social capital on innovation.

The SEARCH (Sharing KnowledgE Assets: InteRregionally Cohesive NeigHborhoods) project financed under the Seventh Framework Programme $(\mathrm{SSH}-2010)^{1}$ aimed to tackle these empirical gaps on ENP in a systematic way. The SEARCH project analysed the impact of the European Neighbourhood Policy on the integration of the EU and its neighbouring countries in terms of their trade and capital flows, mobility and human capital, technological activities and innovation

\footnotetext{
${ }^{1}$ For more details about the results of SEARCH Project, see www.ub.edu/searchproject
} 
diffusion, and institutional environment. The objective of the project was to achieve a better understanding of the conditions characterizing the institutional framework of the ENCs and their economic interactions with the EU in relation to their peoples, capitals, trade, knowledge and innovation. For this purpose, the project was divided into six blocks, analysing the interactions in the flows of goods, productive capital, labour and knowledge among the EU countries and their neighbours.

\section{Papers of the special issue}

This special issue includes 7 papers that cover these different dimensions of the EU-ENCs' relationships. The general purpose of the special issue is to provide a comprehensive overview of the European Neighbourhood Policy by looking specifically at the relationships among the EU and the ENP countries from a variety of economic perspectives, taking also into account the institutional and socio-cultural differences.

Wesselink and Boschma provide a factual description of the history of the European Neighbourhood Policy (ENP), its institutional structure and the policy measures implemented. The paper shows how ENP has matured into a multifaceted policy, and how ENP replaces, or subsumes, a number of previous regional and thematic policies. The ENP is characterised by an interregional element, a broader division into two regional groups (East-South) and includes bilateral agreements. The review concludes that the interregional and regional approaches taken by the ENP have been only weakly developed, given the low intensity of activity and initiative undertaken multilaterally. By contrast, bilateral approaches have been much more actively pursued in several instances.

The objective of the paper by Ramos and Suriñach is to analyse past and future trends in EU-ENCs' bilateral migration relationships. Migration from ENP countries tends to be highly focused on some destination countries because of geographical proximity or strong economic, political and colonialist ties. A gravity model for nearly 200 countries between 1960 and 2010 is estimated to identify the long-run determinants of bilateral migration stocks and is used to obtain medium-run migration forecasts. The results show that an increase in migratory pressures from the ENCs to the EU can be expected in the future. The authors however conclude that it is impossible to account for all factors that can influence migration flows, as illustrated by the 2015 refugee crisis.

Kallioras and Pinna investigate trade activity between the EU and its neighboring countries from 1995 to 2011, employing data derived from the BACI database. Broadly speaking, the ENCs prove to be locked in a traditional inter-industry trade pattern with their more advanced EU partners, casting some doubts on whether this will lead to a narrowing down of the welfare gap between the ENCs and the EU. The paper also shows that the EU-ENCs trade activity has not intensified in relative terms under the ENP framework. Nevertheless, the paper concludes there is potential for the expansion of trade exchange between the EU and its neighboring countries.

The paper by Usai, Autant-Bernard, Gagliardini and Chalaye focuses on knowledge creation in the ENP countries, and knowledge diffusion among both EU and ENP countries. This is the first paper that draws a comprehensive picture of the European Knowledge Neighbourhood, analyzing both input and output indicators in 16 ENP countries. Results show that the relative efficiency in knowledge production is very heterogeneous across countries. Many ENCs show a pervasive weakness in terms of scientific and technological capacity, and knowledge diffusion remains at a 
low level between the EU and its neighbours. However, some ENP countries are pretty efficient in their ability to turn internal and external knowledge into innovation output, pointing to an effective knowledge potential in some parts of the area.

Ascani, Crescenzi and Iammarino examine the geography of foreign investments in the ENP countries. They provide a first investigation of the drivers of FDI in the broadly defined EU neighbourhood, based on data on greenfield investment projects. The different degrees of economic and political integration with the EU, and the recent access of most ENCs to global markets, reflect the large variation in their attractiveness towards foreign capital. Many of these economies remain peripheral in the strategies of MNEs. The results suggest that FDI shows path-dependency and concentration patterns that strengthen core-periphery forces in the EU neighbourhood area.

Bartlett, Čučković and Jurlin look at the institutional quality (e.g. control of corruption, governmental accountability, media freedom) in the European Neighbourhood and investigate whether changes in institutional quality occurred in the period 2004-11. Their analysis reveals differences between ENP countries in the extent of institutional convergence to the EU, in perceptions of corruption, and in the pace of democratic reforms. Analysis of changes in a new index of institutional quality in the public sector reveals a further falling behind the EU. They conclude that convergence targets have not yet been achieved and the outcome is far from certain.

The final contribution by Kaasa also focuses on the institutional dimension but extends it to the analysis of the effect of various cultural dimensions on innovation in the ENP area. The measures of four cultural dimensions following Hofstede were derived from the European and World Value Survey data. The main finding of the paper confirms that innovation is strongly affected by culture: power distance, uncertainty avoidance and masculinity turned out to have a negative effect on innovation performance, while individualism had a positive effect, as expected.

\section{References}

Suriñach, J. (2015) (coordinator). Sharing KnowledgE Assets: InteRregionally Cohesive NeigHborhoods. Final Executive Researh Summary. SEARCH Project 